\title{
Power-laws in economy and finance: some ideas from physics
}

\author{
Jean-Philippe Bouchaud ${ }^{\dagger, *}$ \\ $\dagger$ Science \& Finance, 109-111 rue Victor Hugo, 92532 Levallois CEDEx, FRANCE; \\ http://www.science-finance.fr \\ * Service de Physique de l'État Condensé, Centre d'études de Saclay, \\ Orme des Merisiers, 91191 Gif-sur-Yvette CEDEX, FRANCE
}

May 28, 2018

\begin{abstract}
We discuss several models in order to shed light on the origin of powerlaw distributions and power-law correlations in financial time series. From an empirical point of view, the exponents describing the tails of the price increments distribution and the decay of the volatility correlations are rather robust and suggest universality. However, many of the models that appear naturally (for example, to account for the distribution of wealth) contain some multiplicative noise, which generically leads to non universal exponents. Recent progress in the empirical study of the volatility suggests that the volatility results from some sort of multiplicative cascade. A convincing 'microscopic' (i.e. trader based) model that explains this observation is however not yet available. It would be particularly important to understand the relevance of the pseudo-geometric progression of natural human time scales on the long range nature of the volatility correlations.
\end{abstract}

\section{Introduction}

Physicists are often fascinated by power-laws. The reason for this is that complex, collective phenomenon do give rise to power-laws which are universal, that is to a large degree independent of the microscopic details of the phenomenon. These power-laws emerge from collective action and transcends individual specificities. As such, they are unforgeable signatures of a collective mechanism. Examples in the physics literature are numerous. A well known example is that of phase transitions, where a system evolves from a disordered state to an ordered state: many observables behave as universal power laws in the vicinity of the transition point [1]. This is related to an important property of power-laws, 
namely scale invariance the characteristic length scale of a physical system at its critical point is infinite, leading to self-similar, scale-free fluctuations. Another example is fluid turbulence, where the statistics of the velocity field has scale invariant properties, to a large extent independent of the nature of the fluid, of the power injected, etc. [2].

Power-laws are also often observed in economic and financial data [3, 4, 5 , 6. [7. Compared to physics, however, much less effort has been devoted to understand these power-laws in terms of 'microscopic' (i.e. agent based) models and to relate the value of the exponents to generic mechanisms. The aim of this contribution is to give a short review of diverse power-laws observed in economy/finance, and to discuss several simple models (most of them inspired from physics) which naturally lead to power-laws and could serve as a starting point for further developments. It should be stressed that none of the models presented here are intended to be fully realistic and complete, but are of pedagogical interest: they nicely illustrate how and when power-laws can arise. This paper is furthermore written in the spirit of a conference proceedings, and many rather speculative ideas are put forward to fuel further discussions.

\section{Empirical power-laws: a short review}

\subsection{Distributional power-laws}

The oldest and most famous power-law in economy is the Pareto distribution of wealth [3]. The distribution of individual wealths $P(W)$ is often described, in its asymptotic tail, by a power law:

$$
P(W) \simeq \frac{W_{0}^{\mu}}{W^{1+\mu}} \quad W \gg W_{0}
$$

where $\mu$ characterize the decay of the distribution for large $W$ 's: the smaller the value of $\mu$, the slower the decay, and the larger the contrast between the richest and the poorest. More precisely, in a Pareto population of size $N$, the ratio of the largest wealth to the typical (e.g. median) wealth grows as $N^{1 / \mu}$. In the case $\mu<1$, the average wealth diverges: this corresponds to an economy where a finite fraction of the total wealth is in the hands of a few individuals, even when $N \rightarrow \infty$. On the contrary, when $\mu>1$, the richest individual only holds a zero fraction of the total wealth (again in the limit $N \rightarrow \infty$ ). Empirically, the exponent $\mu$ is in the range $1-2$. This Pareto tail also describes the distribution of income, the size of companies, of pension funds, etc. [8, 9, 10].

In financial markets, the distribution of the price increments $\delta x_{t}=x\left(t^{\prime}\right)-$ $x(t)$ over different time scales $t^{\prime}-t$ is important both for risk control purposes

\footnotetext{
${ }^{1}$ Power-law distributions are scale invariant in the sense that the relative probability to observe an event of a given size and an event ten times larger is independent of the reference scale.
} 
and for derivative pricing models [11]. The availability of long series of high frequency data has motivated many empirical studies in the past few years. By pooling together the statistics of a thousand U.S. stocks, it is possible to study quite accurately the far tail of the distribution of intra-day price increments $\delta x$, which can be fitted as a power law [12]:

$$
P(\delta x) \simeq \frac{\delta x_{0}^{\mu}}{|\delta x|^{1+\mu}},
$$

with $\mu$ in found to be close to 3. Similar values have also been reported for Japanese stocks [12], German stocks [13], currencies [14, 15], bond markets [16], and perhaps also the distribution of the (time dependent) daily volatility $\sigma$, defined as an average over high frequency returns 17] (although other works report a log-normal distribution [22, 25]). 2 This suggests that the value of $\mu$ could be universal. Note however that the value of $\mu$ depends somewhat on the stock and on the period of time studied. For example, the value of $\mu$ for the S\&P500 during the years 1991-1995 is found to be close to 5. Furthermore, as the time lag used for the definition of $\delta x$ increases, the effective exponent describing the tail of the distribution increases as the distribution becomes more and more Gaussian like [11, 12].

\subsection{Temporal power-laws}

Actually, the price increment at time $t$ can usefully be thought of as the product of a sign part $\epsilon_{t}$ and an amplitude part (or volatility) $\sigma_{t}$ :

$$
\delta x_{t}=\epsilon_{t} \times \sigma_{t} .
$$

The random variable $\epsilon_{t}$ has short ranged temporal correlations, extending over a few minutes or so on liquid markets $[11,[6]$. The volatility, on the contrary, has very long ranged correlations, which can be fitted as a power-law with a small exponent $\nu$ 18, 19, 14, 20, 21, 22:

$$
C_{1}(\tau)=\left\langle\sigma_{t} \sigma_{t+\tau}\right\rangle-\left\langle\sigma_{t}\right\rangle^{2} \simeq \frac{c_{1}}{\tau^{\nu}},
$$

with $\nu$ approximately equal to 0.1. This behaviour is, again, seen on many different types of markets, and quantifies the intermittent activity of these markets: volatility tends to cluster in bursts which persist over very different time scales, from minutes to months. A similar power-law behaviour of the temporal correlation of the volume of transactions (number of trades) is also observed [23, 17]. This is not surprising, since volatility and volume are strongly correlated. From a empirical point of view, the intermittent nature of the activity in financial markets is similar to the energy dissipation in a turbulent fluid [2, 24].

\footnotetext{
${ }^{2}$ Note that the tails of a log-normal distribution can be fitted (over a restricted interval) by a power-law. Therefore it is not always easy to distinguish between true power-laws and effective power-laws.
} 
In fact, the distribution of $\log \sigma$ is not far from being Gaussian [22, 25]. Therefore, it is natural to study the temporal correlation of $\log \sigma$ [26, 27]:

$$
C_{0}(\tau)=\left\langle\log \sigma_{t} \log \sigma_{t+\tau}\right\rangle-\left\langle\log \sigma_{t}\right\rangle^{2} .
$$

This correlation is also found to decay very slowly with $\tau$. This decay can be fitted by a logarithm [26, 27]: $C_{0}(\tau)=\lambda^{2} \log (T / \tau)$, with a rather small value of $\lambda^{2} \simeq 0.05$. This, together with the assumption that $\log \sigma$ is exactly Gaussian, leads to a strict multifractal model for the price changes [27], in the sense that different moments of the price increments scale with different powers of time [24, 28, 29]. Within this model, one finds 27]:

$$
\left\langle|x(t+\tau)-x(t)|^{q}\right\rangle \propto \tau^{\zeta_{q}} \quad \zeta_{q}=\frac{q}{2}\left[1-\lambda^{2}(q-2)\right],
$$

for $\tau \ll T$ and $q \lambda^{2}<1$ (for higher values of $q$, the corresponding moment is divergent). It is easy to show that in this model, the exponent $\nu$ defined by Eq. (41) is equal to $\lambda^{2}$. On the other hand, one can also fit $C_{0}(\tau)$ by a power-law with a small exponent, in which case the model would only be approximately multifractal, in the sense that the quantity $\left\langle|x(t+\tau)-x(t)|^{q}\right\rangle$ is the sum of different powers of $\tau$, which can also be fitted by an effective, $q$-dependent exponent $\zeta_{q}<q / 2[30]$.

\section{Simple models of wealth distribution}

\subsection{A model with trading and speculative investment}

As a simple dynamical model of economy, one can consider a stochastic equation for the wealth $W_{i}(t)$ of the $i^{t h}$ agent at time $t$, that takes into account the exchange of wealth between individuals through trading, and is consistent with the basic symmetry of the problem under a change of monetary units. Since the unit of money is arbitrary, one indeed expects that the equation governing the evolution of wealth should be invariant when all $W_{i}$ 's are multiplied by a common (arbitrary) factor. A rather general class of equation which has this property is the following [31, 32]:

$$
\frac{d W_{i}}{d t}=\eta_{i}(t) W_{i}+\sum_{j(\neq i)} J_{i j} W_{j}-\sum_{j(\neq i)} J_{j i} W_{i},
$$

where $\eta_{i}(t)$ is a Gaussian random variable of mean $m$ and variance $2 \sigma^{2}$, which describes the spontaneous growth or decrease of wealth due to investment in stock markets, housing, etc. The term involving the (asymmetric) matrix $J_{i j}$

describes the amount of money that agent $j$ spends buying the production of agent $i$. We assume that this production is consumable, and therefore must not 
be counted as part of the wealth of $i$ once it is bought. The equation ( 7 is obviously invariant under the scale transformation $W_{i} \rightarrow \lambda W_{i}$.

The simplest trading network one can think of is when all agents exchange with all others at the same rate, i.e $J_{i j} \equiv J / N$ for all $i \neq j$. Here, $N$ is the total number of agents, and the scaling $J / N$ is needed to make the limit $N \rightarrow \infty$ well defined. In this case, the equation for $W_{i}(t)$ becomes:

$$
\frac{d W_{i}}{d t}=\eta_{i}(t) W_{i}+J\left(\bar{W}-W_{i}\right)
$$

where $\bar{W}=N^{-1} \sum_{i} W_{i}$ is the average overall wealth. This is called, in the physics language, a 'mean-field' model since all agents feel the very same influence of their environment. It is useful to rewrite eq. (8) in terms of the normalised wealths $w_{i} \equiv W_{i} / \bar{W}$. This leads to:

$$
\frac{d w_{i}}{d t}=\left(\eta_{i}(t)-m-\sigma^{2}\right) w_{i}+J\left(1-w_{i}\right)
$$

to which one can associate the following Fokker-Planck equation for the evolution of the density of wealth $P(w, t)$ :

$$
\frac{\partial P}{\partial t}=\frac{\partial\left[J(w-1)+\sigma^{2} w\right] P}{\partial w}+\sigma^{2} \frac{\partial}{\partial w}\left[w \frac{\partial w P}{\partial w}\right] .
$$

The equilibrium, long time solution of this equation is easily shown to be:

$$
P_{e q}(w)=\frac{(\mu-1)^{\mu}}{\Gamma[\mu]} \frac{e^{-\frac{\mu-1}{w}}}{w^{1+\mu}} \quad \mu \equiv 1+\frac{J}{\sigma^{2}} .
$$

Therefore, one finds in this model that the distribution of wealth indeed exhibits a Pareto power-law tail for large w's. Interestingly, however, the value of the exponent $\mu$ is not universal, and depends on the parameter of the model $\left(J\right.$ and $\sigma^{2}$, but not on the average growth rate $m$ ). In agreement with intuition, the exponent $\mu$ grows (corresponding to a narrower distribution), when exchange between agents is more active (i.e. when $J$ increases), and also when the success in individual investment strategies is more narrowly distributed (i.e. when $\sigma^{2}$ decreases). In this model, the exponent $\mu$ is always found to be larger than one. This means that the wealth is not too unevenly distributed within the population.

Let us now describe more realistic trading network, where the number of economic neighbours to a given individual is finite. We will assume that the matrix $J_{i j}$ is still symmetrical, and is either equal to $J$ (if $i$ and $j$ trade), or equal to 0 . A reasonable assumption is that the graph describing the connectivity of the population is completely random, i.e. that two points are neighbours with probability $c / N$ and disconnected with probability $1-c / N$. In such a graph, the average number of neighbours is equal to $c$. We have performed some numerical 
simulations of Eq. (7) for $c=4$ and have found that the wealth distribution still has a power-law tail, with an exponent $\mu$ which only depends on the ratio $J / \sigma^{2}$. However, one finds that the exponent $\mu$ can now be smaller than one for sufficiently small values of $J / \sigma^{2}$ [31]. In this model, one therefore expects 'wealth condensation' when the exchange rate is too small, in the sense that a finite fraction of the total wealth is held by only a few individuals.

Although not very realistic, one could also think that the individuals are located on the nodes of a d-dimensional hyper-cubic lattice, trading with their neighbours up to a finite distance. In this case, one knows that for $d>2$ there exists again a phase transition between a 'social' economy where $\mu>1$ and a rich dominated phase $\mu<1$. On the other hand, for $d \leq 2$, and for large populations, one is always in the extreme case where $\mu \rightarrow 0$ at large times. In the case $d=1$, i.e. operators organized along a chain-like structure (as a simple model of intermediaries), one can actually compute exactly the distribution of wealth [33, 34]. One finds for example that the ratio of the maximum wealth to the typical (e.g. median) wealth behaves as $\exp \sqrt{N}$, where $N$ is the size of the population, instead of $N^{1 / \mu}$ in the case of a Pareto distribution with $\mu>0$.

The conclusion of the above results is that the distribution of wealth tends to be very broadly distributed when exchanges are limited, either in amplitude (i.e. $J$ too small compared to $\sigma^{2}$ ) or topologically (a $\mathrm{s}$ in the above chain structure). Favoring exchanges (in particular with distant neighbours) seems to be an efficient way to reduce inequalities.

\subsection{Two related models}

Let us now interpret $W_{i}$ as the size of a company. The growth of this company can take place either from internal growth, depending on its success or failure. This leads to a term $\eta_{i}(t) W_{i}$ much as above. Another possibility is merging with another company. If the merging process between two companies is completely random and takes place at a rate $\Gamma$ per unit time, then the model is exactly the same as the one considered by Derrida and Spohn [35] in the context of 'directed polymers in random media', and bears strong similarities with the model discussed in the previous section. One again finds that the distribution of $W$ 's is a power-law with a non universal exponent, which depends on the values of $\Gamma$ and $\sigma^{2}$, and can be smaller than one.[]

One can also consider a model where companies grow at a random rate $\eta$, but may also suddenly die at a rate $\Gamma^{\prime}$ per unit time, and be replaced by a new (small) company. There again, one finds a stationary Pareto distribution, with a non universal exponent which depends continuously on $m, \sigma$ and $\Gamma^{\prime}$.

\footnotetext{
${ }^{3}$ In the language of disordered systems, this corresponds to the 'glassy' phase of the directed polymer, where the partition function is dominated by a few paths only.
} 


\section{Simple models for herding and copy-cats}

We now turn to simple models for thick tails in the distribution of price increments in financial markets. An intuitive explanation is herding: if a large number of agents acting on markets coordinate their action, the price change is likely to be huge due to a large unbalance between buy and sell orders [36]. However, this coordination can result from two rather different mechanisms.

- One is the feedback of past price changes onto themselves, which we will discuss in the following section. Since all agents are influenced by the very same price changes, this can induce non trivial collective behaviour: for example, an accidental price drop can trigger large sell orders, which lead to further downward moves.

- The second is direct influence between the traders, through exchange of information that leads to 'clusters' of agents sharing the same decision to buy, sell, or be inactive at any given instant of time.

\subsection{Herding and percolation}

A simple model of how herding affects the price fluctuations was proposed in [37]. It assumes that the price increment $\delta x$ depends linearly on the instantaneous offset between supply and demand [37, 38]. More precisely, if each operator in the market $i$ wants to buy or sell a certain fixed quantity of the financial asset, one has [37: :

$$
\delta x=\frac{1}{\lambda} \sum_{i} \varphi_{i},
$$

where $\varphi_{i}$ can take the values $-1,0$ or +1 , depending on whether the operator $i$ is selling, inactive, or buying, and $\lambda$ is a measure of the market depth. Note that the linearity of this relation, even for small arguments, has been questioned by Zhang [39]. Suppose now that the operators interact among themselves in an heterogeneous manner: with a small probability $c / N$ (where $N$ is the total number of operators on the market), two operators $i$ and $j$ are 'connected', and with probability $1-c / N$, they ignore each other. The factor $1 / N$ means that on average, the number of operator connected to any particular one is equal to $c$ (the resulting graph is precisely the same as the random trading graph of Section 3.1). Suppose finally that if two operators are connected, they come to agree on the strategy they should follow, i.e. $\varphi_{i}=\varphi_{j}$.

It is easy to understand that the population of operators clusters into groups sharing the same opinion. These clusters are defined such that there exists a connection between any two operators belonging to this cluster, although the

\footnotetext{
${ }^{4}$ This can alternatively be written for the relative price increment $\delta x / x$, which is more adapted to describe long time scales. On short time scales, however, an additive model is preferable. See the discussion in [11].
} 
connection can be indirect and follow a certain 'path' between operators. These clusters do not have all the same size, i.e. do not contain the same number of operators. If the size of cluster $\mathcal{C}$ is called $S(\mathcal{C})$, one can write:

$$
\delta x=\frac{1}{\lambda} \sum_{\mathcal{C}} S(\mathcal{C}) \varphi(\mathcal{C})
$$

where $\varphi(\mathcal{C})$ is the common opinion of all operators belonging to $\mathcal{C}$. The statistics of the price increments $\delta x$ therefore reduces to the statistics of the size of clusters, a classical problem in percolation theory [40]. One finds that as long as $c<1$ (less than one 'neighbour' on average with whom one can exchange information), then all $S(\mathcal{C})$ 's are small compared to the total number of traders $N$. More precisely, the distribution of cluster sizes takes the following form in the limit where $1-c=\epsilon \ll 1$ :

$$
P(S) \propto_{S \gg 1} \frac{1}{S^{5 / 2}} \exp -\epsilon^{2} S \quad S \ll N .
$$

When $c=1$ (percolation threshold), the distribution becomes a pure power-law with an exponent $\mu=3 / 2$, and the Central Limit Theorem tells us that in this case, the distribution of the price increments $\delta x$ is precisely a pure symmetrical Lévy distribution of index $\mu=3 / 2$ [1] (assuming that $\varphi= \pm 1$ play identical roles, that is if there is no global bias pushing the price up or down). If $c<1$, on the other hand, one finds that the Lévy distribution is truncated exponentially, leading to a larger effective tail exponent $\mu$ 37]. If $c>1$, a finite fraction of the $N$ traders have the same opinion: this leads to a crash.

\subsection{Avalanches of opinion changes}

The above model is interesting but has one major drawback: one has to assume that the parameter $c$ is smaller than one, but relatively close to one such that Eq. (14) is valid, and non trivial statistics follows. One should thus explain why the value of $c$ spontaneously stabilises in the neighbourhood of the critical value $c=1$. Furthermore, this model is purely static, and does not specify how the above clusters evolve with time. As such, it cannot address the problem of volatility clustering. Several extensions of this simple model have been proposed [41, 42], in particular to increase the value of $\mu$ from $\mu=3 / 2$ to $\mu \sim 3$ and to account for volatility clustering.

One particularly interesting model is inspired by the recent work of Dahmen and Sethna [43], that describes the behaviour of random magnets in a time dependent magnetic field. Transposed to the present problem (as first suggested in [11), this model describes the collective behaviour of a set of traders exchanging information, but having all different a priori opinions. One trader can however change his mind and take the opinion of his neighbours if the coupling is strong, or if the strength of his a priori opinion is weak. More precisely, the 
opinion $\varphi_{i}(t)$ of agent $i$ at time $t$ is determined as:

$$
\varphi_{i}(t)=\operatorname{sign}\left(h_{i}(t)+\sum_{j=1}^{N} J_{i j} \varphi_{j}(t)\right),
$$

where $J_{i j}$ is a connectivity matrix describing how strongly agent $j$ affects agent $i$, and $h_{i}(t)$ describes the a priori opinion of agent $i: h_{i}>0$ means a propensity to buy, $h_{i}<0$ a propensity to sell. We assume that $h_{i}$ is a random variable with a time dependent mean $\bar{h}(t)$ and root mean square $\Delta$. The quantity $\bar{h}(t)$ represents for example confidence in long term economy growth $(\bar{h}(t)>0)$, or fear of recession $(\bar{h}(t)<0$, leading to a non zero average pessimism or optimism. Suppose that one starts at $t=0$ from a 'euphoric' state, where $\bar{h} \gg \Delta, J$, such that $\varphi_{i}=1$ for all $i$ 's. f Now, confidence is decreased progressively. How will sell orders appear?

Interestingly, one finds that for small enough influence (or strong heterogeneities of agents' anticipations), i.e. for $J \ll \Delta$, the average opinion $O(t)=$ $\sum_{i} \varphi_{i}(t) / N$ evolves continuously from $\left.O(t=0)\right)=+1$ to $O(t \rightarrow \infty)=-1$. The situation changes when imitation is stronger since a discontinuity then appears in $O(t)$ around a 'crash' time $t_{c}$, when a finite fraction of the population simultaneously change opinion. The gap $O\left(t_{c}^{-}\right)-O\left(t_{c}^{+}\right)$opens continuously as $J$ crosses a critical value $J_{c}(\Delta)$ [43]. For $J$ close to $J_{c}$, one finds that the sell orders again organise as avalanches of various sizes, distributed as a power-law with an exponential cut-off. In the 'mean-field' case where $J_{i j} \equiv J / N$ for all $i j$, one finds $\mu=5 / 4$. Note that in this case, the value of the exponent $\mu$ is universal, and does not depend, for example, on the shape of the distribution of the $h_{i}$ 's, but only on some global properties of the connectivity matrix $J_{i j}$.

A slowly oscillating $\bar{h}(t)$ can therefore lead to a succession of bull and bear markets, with a strongly non Gaussian, intermittent behaviour, since most of the activity is concentrated around the crash times $t_{c}$. Some modifications of this model are however needed to account for the empirical value $\mu \sim 3$ observed on the distribution of price increments (see the discussion in 414).

Note that the same model can be applied to other interesting situations, for example to describe how applause end in a concert hall [44] (here, $\varphi= \pm 1$ describes, respectively, a clapping and a quiet person, and $O(t)$ is the total clapping activity). Clapping can end abruptly (as often observed, at least by the present author) when imitation is strong, or smoothly when many fans are present in the audience. A static version of the same model has been proposed to describe rational group decision making [45].

\footnotetext{
${ }^{5}$ Here $J$ denotes the order of magnitude of $\sum_{j} J_{i j}$
} 


\section{Models of feedback effects on price fluctua- tions}

\subsection{Risk-aversion induced crashes}

The above average 'stimulus' $\bar{h}(t)$ may also strongly depend on the past behaviour of the price itself. For example, past positive trends are, for many investors, incentives to buy, and vice-versa. Actually, for a given trend amplitude, price drops tend to feedback more strongly on investors' behaviour than price rises. Risk-aversion creates an asymmetry between positive and negative price changes [46]. This is reflected by option markets, and pushes the price of out-of-the-money puts up.

Similarly, past periods of high volatility increases the risk of investing in stocks, and usual portfolio theories then suggest that sell orders should follow. A simple mathematical transcription of these effects is to write Eq. (12) in a linearized, continuous time form:

$$
\frac{d x}{d t} \equiv u=\frac{1}{\lambda} \bar{h}(t)
$$

and write a dynamical equation for $\bar{h}(t)$ which encodes the above feedback effects [46, 38]:

$$
\frac{d \bar{h}}{d t}=a u-b u^{2}-c \bar{h}+\eta(t)
$$

where $a$ describes trends following effects, $b$ risk aversion effects (breaking the $u \rightarrow-u$ symmetry), $c$ is a mean reverting term which arises from market clearing mechanisms, and $\eta$ is a noise term representing random external news [46]. Eliminating $\bar{h}$ from the above equations leads to:

$$
\frac{d u}{d t}=-\gamma u-\beta u^{2}+\frac{1}{\lambda} \eta(t) \equiv-\frac{\partial V}{\partial u}+\frac{1}{\lambda} \eta(t)
$$

where $\gamma=c-a / \lambda$ and $\beta=b / \lambda$. Equation (18) represents the evolution of the position $u$ of a viscous fictitious particle in a 'potential' $V(u)=\gamma u^{2} / 2+\beta u^{3} / 3$. If trend following effects are not too strong, $\gamma$ is positive and $V(u)$ has a local minimum for $u=0$, and a local maximum for $u^{*}=-\gamma / \beta$, beyond which the potential plummets to $-\infty$.1] A 'potential barrier' $V^{*}=\gamma u^{* 2} / 6$ separates the (meta-)stable region around $u=0$ from the unstable region. The nature of the motion of $u$ in such a potential is the following: starting at $u=0$, the particle has a random harmonic-like motion in the vicinity of $u=0$ until an 'activated' event (i.e. driven by the noise term) brings the particle near $u^{*}$.

\footnotetext{
${ }^{6}$ In the following, the herding effects described by $J_{i j}$ are neglected, or more precisely, only their average effect encoded by $\bar{h}$ is taken into account.

${ }^{7}$ If $\gamma$ is negative, the minimum appears for a positive value of the return $u^{*}$. This corresponds to a speculative bubble. See 46.
} 
Once this barrier is crossed, the fictitious particle reaches $-\infty$ in finite time. In financial terms, the regime where $u$ oscillates around $u=0$ and where $\beta$ can be neglected, is the 'normal' fluctuation regime. This normal regime can however be interrupted by 'crashes', where the time derivative of the price becomes very large and negative, due to the risk aversion term $b$ which destabilizes the price by amplifying the sell orders. The interesting point is that these two regimes can be clearly separated since the average time $t^{*}$ needed for such crashes to occur is exponentially large in $V^{*}$ [47, and can thus appear only very rarely. A very long time scale is therefore naturally generated in this model.

Note that in this line of thought, a crash occurs because of an improbable succession of unfavorable events, and not due to a single large event in particular. Furthermore, there are no 'precursors' in this model: before $u$ has reached $u^{*}$, it is impossible to decide whether it will do so or whether it will quietly come back in the 'normal' region $u \simeq 0$. Solving the Fokker-Planck equation associated to the Langevin equation (18) leads to a stationary state with a power law tail for the distribution of $u$ (i.e. of the instantaneous price increment) decaying as $u^{-2}$ for $u \rightarrow-\infty$. More generally, if the risk aversion term took the form $-b u^{1+\mu}$, the negative tail would decay as $u^{-1-\mu}$.

\subsection{Dynamical volatility models}

The simplest model that describes volatility feedback effects is to write an ARCH like equation [48], which relates today's activity to a measure of yesterday's activity, for example:

$$
\sigma_{k}=\sigma_{k-1}+K\left(\sigma_{0}-\sigma_{k-1}\right)+g\left|\delta x_{k-1}\right|
$$

where $\sigma_{0}$ is an average volatility level, $K$ a mean-reverting term, and $g$ describes how much yesterday's observed price change affects the behaviour of traders today. Since $\left|\delta x_{k-1}\right|$ is a noisy version of $\sigma_{k-1}$, the above equation is, in the continuous time limit, a Langevin equation with multiplicative noise:

$$
\frac{d \sigma}{d t}=K\left(\sigma_{0}-\sigma\right)+g \sigma \eta(t),
$$

which is, up to notation changes, exactly the same equation as (9) above. Therefore, the stationary distribution of the volatility in this model is again given by Eq. (11), with the tail exponent now given by $\mu-1 \propto K / g^{2}$ : over-reactions to past informations (i.e. large values of $g$ ) decreases the tail exponent $\mu$. Therefore, one again finds a non universal exponent in this model, which is bequeathed to the distribution of price increments if one assumes that the 'sign' contribution to $\delta x_{k}$ (see Eq. (30) has thin tails.

Note that the temporal correlations of the volatility $\sigma$ can be exactly calculated within this model [49], and is found to be exponentially decaying, at variance with the slow power-law (or logarithmic) decay observed empirically. 
Furthermore, the distribution (11) does not concur with the nearly log-normal distribution of the volatility that seems to hold empirically [22, 25].

At this point, the slow decay of the volatility can be ascribed to two rather different mechanisms. One is the existence of traders with many different time horizons, as suggested in 50, 21. If traders are affected not only by yesterday's price change amplitude $\left|\delta x_{k-1}\right|$, but also by price changes on coarser time scales $\left|x_{k}-x_{k-p}\right|$, then the correlation function is expected to be a sum of exponentials with decay rates given by $p^{-1}$. Interestingly, if the different $p$ 's are uniformly distributed on a log scale, the resulting sum of exponentials is to a good approximation decaying as a logarithm. More precisely 8

$$
C(\tau)=\frac{1}{\log \left(p_{\max } / p_{\min }\right)} \int_{p_{\min }}^{p_{\max }} d(\log p) \exp (-\tau / p) \simeq \frac{\log \left(p_{\max } / \tau\right)}{\log \left(p_{\max } / p_{\min }\right)},
$$

whenever $p_{\min } \ll \tau \ll p_{\max }$. Now, the human time scales are indeed in a natural pseudo-geometric progression: hour, day, week, month, trimester, year 21.

Yet, some recent numerical simulations of traders allowed to switch between different strategies (active/inactive, or chartist/fundamentalist) suggest strongly intermittent behaviour [51, 42, 52], and a slow decay of the volatility correlation function without the explicit existence of logarithmically distributed time scales. An intuitive, semi-analytical explanation of this numerical finding is however still lacking. Note that from a purely phenomenological point of view, one can define a model that assumes that the volatility today is the retarded result of past influences:

$$
\sigma_{k}=\sum_{p=0}^{\infty} M(p) \eta_{k-p},
$$

where the $\eta$ 's are uncorrelated shocks and $M(p)$ a memory kernel describing how much the past is remembered. If one chooses a power-law decay for $M(p)$ with an exponent $\alpha$, then the decay of the correlation function of $\sigma$ is also a power-law with an exponent $\nu$ (defined in Eq. (位) given by $\nu=2 \alpha-1$. The value $\nu=0$ corresponds to a memory kernel decaying as $1 / \sqrt{p}$, i.e., as the probability of not returning to the origin for a random walk of length $p$. Whether this is a mere coincidence is left for future investigations. Note however that a decomposition such as (22) naturally leads to a normal distribution for $\sigma$, very different from the empirical log-normal behaviour of the volatility. A consistent market model leading simultaneously to a nearly log-normal distribution and a nearly logarithmic (in time) decay of the volatility correlation remains to be built. In this respect, the cascade construction of Mandelbrot et al. 28] does indeed have these two properties exactly, but is non-causal (the volatility today depends on future events) and lacks an intuitive interpretation.

\footnotetext{
${ }^{8}$ This mechanism is well known in the physics of slow, glassy systems, where the relaxation times $p$ are the exponential of some local activation energy $E$. At low temperatures, any small dispersion of $E$ will generate a $1 / p$ distribution for $p$ over a wide time interval, and eventually to a logarithmic relaxation.
} 


\section{Concluding remarks}

Many ideas have been presented in this rather hairy paper. Most of them will perhaps turn out to be wrong, but will hopefully motivate some further work to understand the origin of power-law distributions and power-law correlations in financial time series. From an empirical point of view, the exponents describing the tails of the price increments distribution and the decay of the volatility correlations are rather robust and suggest some kind of universality, probably related to the fact that all speculative markets obey common rules where simple human psychology (greed and fear) coupled to basic mechanisms of price formation ultimately lead to the emergence of scaling and power-laws. Still, many points remain obscure: we have seen above that models that appear naturally in the context of economy and finance contain multiplicative noise, which is a simple mechanism for power-law distributions (as emphasized in, e.g. [32, 41]). However, these models generically lead to non universal exponents (as discussed above in the context of the Pareto tails) that depends continuously on the value of the parameters. Recent progress in the empirical study of the volatility (using, e.g., wavelets [26, 27]) suggests that the volatility results from some sort of multiplicative cascade, as postulated in [28]. A convincing 'microscopic' (i.e. trader based) model that explains this observation would at this stage be very valuable, and would shed light on the possible relevance of the pseudo geometric human time scales on the decay of the volatility correlations.

\section{Acknowledgments}

I want to warmly thank all my collaborators for sharing with me their knowledge: P. Cizeau, R. Cont, I. Giardina, L. Laloux, A. Matacz, M. Mézard, M. Meyer and M. Potters. Several discussions with E. Bacry, R. da Silvera, M. Dacorogna, D. Farmer, T. Lux, R. Mantegna, J.F. Muzy, G. Stanley, D. Stauffer and S. Solomon have been very useful.

\section{References}

[1] see e.g. N. Goldenfeld, Lectures on phase transitions and critical phenomena, Frontiers in Physics, 1992, for an excellent introduction.

[2] U. Frisch, Turbulence: The Legacy of A. Kolmogorov, Cambridge University Press (1997).

[3] V. Pareto, Cours d'économie politique. Reprinted as a volume of Oeuvres Complètes (Droz, Geneva, 1896-1965).

[4] B.B. Mandelbrot, Int. Eco. Rev. 1 (1960) 79, B. B. Mandelbrot, Journal of Business 36, 394 (1963); ibid. 40, 394 (1967). 
[5] B.B. Mandelbrot, Fractals and Scaling in Finance, Springer (1997).

[6] R. Mantegna \& H. E. Stanley, An introduction to Econophysics, Cambridge University Press, 1999.

[7] J. D. Farmer, Physicists attempt to scale the ivory towers of finance, in Computing in Science and Engineering, November 1999.

[8] A. B. Atkinson, A. J. Harrison, Distribution of total wealth in Britain (Cambridge University Press, 1978), Y. Ijri, H. A. Simon, Skew distribution of sizes of Business Firms (North-Holland, Amsterdam),

[9] M. H. R. Stanley, S. Buldyrev, S. Havlin, R. Mantegna, M. Salinger, H. E. Stanley, Eco. Lett. 49 (1995) 453.

[10] H. Aoyama, Y. Nagahara, M. Okasaki, W. Souma, H. Takayasu, M. Takayasu, Pareto's law for income of individuals and debt of bankrupt companies, e-print cond-mat/0006038.

[11] J.-P. Bouchaud and M. Potters, Théorie des Risques Financiers, AléaSaclay, 1997; Theory of Financial Risks, Cambridge University Press, 2000.

[12] V. Plerou, P. Gopikrishnan, L.A. Amaral, M. Meyer, H.E. Stanley, Phys. Rev. E60 6519 (1999).

[13] T. Lux, Applied Financial Economics, 6, 463, (1996).

[14] M. M. Dacorogna, U. A. Müller, R. J. Nagler, R. B. Olsen and O. V. Pictet, J. Inter. Money and Finance 12, 413 (1993); D. M. Guillaume, M. M. Dacorogna, R. D. Davé, U. A. Müller, R. B. Olsen and O. V. Pictet, Finance and Stochastics 195 (1997).

[15] F. Longin, Journal of Business, 69383 (1996)

[16] J. Nuyts, I. Platten, Phenomenology of the term structure of interest rates with Padé approximants, e-print cond-mat/9901096.

[17] V. Plerou, P. Gopikrishnan, L.A. Amaral, X. Gabaix, H.E. Stanley, e-print cond-mat/9912051.

[18] A. Lo, Econometrica, 59, 1279 (1991).

[19] Z. Ding, C. W. J. Granger and R. F. Engle, J. Empirical Finance 1, 83 (1993).

[20] R. Cont, M. Potters, J.-P. Bouchaud, Scaling in stock market data: stable laws and beyond, in Scale invariance and beyond, B. Dubrulle, F. Graner, D. Sornette (Edts.), EDP Sciences (1997). 
[21] M. Potters, R. Cont, J.-P. Bouchaud, Europhys. Lett. 41, 239 (1998).

[22] Y. Liu, P. Cizeau, M. Meyer, C.-K. Peng, H. E. Stanley, Physica A245 437 (1997), P. Cizeau, Y. Liu, M. Meyer, C.-K. Peng, H. E. Stanley, Physica A245 441 (1997).

[23] G. Bonnano, F. Lillo, R. Mantegna, Dynamics of the number of trades in financial securities, e-print cond-mat/9912006

[24] The first paper to suggest this analogy is S. Ghashghaie, W. Breymann, J. Peinke, P. Talkner, Y. Dodge, Nature 381767 (1996). See however [6, 26, 27], and A. Arneodo, J.P. Bouchaud, R. Cont, J.F. Muzy, M. Potters, D. Sornette, cond-mat/9607120, unpublished.

[25] The distribution of individual S\&P stock volatilities is also found to be nearly log-normal: Science \& Finance unpublished report, (2000).

[26] A. Arnéodo, J.-F. Muzy, D. Sornette, Eur. Phys. J. B 2, 277 (1998)

[27] see in particular the exciting work of J.-F. Muzy, J. Delour, E. Bacry, e-print cond-mat/0005400.

[28] A. Fisher, L. Calvet, B.B. Mandelbrot, 'Multifractality of DEM/\$ rates', Cowles Foundation Discussion Paper 1165; B.B. Mandelbrot, Scientific American, Feb. (1999).

[29] F. Schmitt, D. Schertzer, S. Lovejoy, Applied Stochastic Models and Data Analysis, 1529 (1999); M.-E. Brachet, E. Taflin, J.M. Tchéou, Scaling transformation and probability distributions for financial time series, eprint cond-mat/9905169

[30] J.P. Bouchaud, M. Potters, M. Meyer, Eur. Phys. J. B 13, 595 (1999).

[31] J.P. Bouchaud, M. Mézard, Physica A, 282, 536 (2000).

[32] Similar models were discussed in: S. Solomon, in Computational Finance 97, eds. A-P. N. Refenes, A.N. Burgess, J.E. Moody (Kluwer Academic Publishers 1998); cond-mat/9803367; O. Malcai O. Biham and S. Solomon, Phys. Rev. E, 60, 1299 (1999); M. Marsili, S. Maslov, Y.C. Zhang, Physica A 253, 403 (1998)

[33] D.S. Fisher, C. Henley, D.A. Huse, Phys. Rev. Lett. 552924 (1985).

[34] I. Giardina, J.P. Bouchaud, M. Mézard, Population dynamics in a random environment, cond-mat/0005187

[35] B. Derrida, H. Spohn, J. Stat. Phys. 51 (1988) 817 
[36] For an introduction and references, see the very interesting book of R. Schiller, Irrational Exuberance, Princeton University Press (2000).

[37] R. Cont, J.P. Bouchaud, Macroeconomics Dynamics, 4, 170 (2000). See the numerous references of this paper for other works on herding in economy and finance.

[38] J.D. Farmer, Market Force, Ecology and Evolution, e-print adaporg/9812005.

[39] Y.C. Zhang, Physica A269 30 (1999).

[40] D. Stauffer, A. Aharony, Introduction to percolation, Taylor-Francis, London, 1994.

[41] D. Sornette, D. Stauffer, H. Takayasu, Market fluctuations, multiplicative and percolation models, size effects and predictions, e-print condmat/9909439, and references therein.

[42] G. Iori, A microsimulation of traders activity in the stock market: the role of heterogeneity, agents' interactions and trade frictions, e-print adaporg/9905005.

[43] K. Dahmen, J. P. Sethna, Phys. Rev. B 53, 14872 (1996).

[44] R. da Silvera, J.P. Bouchaud, in preparation.

[45] S. Galam, Physica A238, 66 (1997).

[46] J.-P. Bouchaud, R. Cont, European Journal of Physics B 6, 543 (1998).

[47] S. Chandraseckar, Rev. Mod. Phys. 151 (1943), reprinted in 'Selected papers on noise and stochastic processes', Dover (1954).

[48] see, e.g. C. Gourieroux, A. Montfort, Statistics and Econometric Models, Cambridge University Press, 1996.

[49] R. Graham, A. Schenzle, Phys. Rev. A 25 (1982) 1731.

[50] U.A. Muller, M.M. Dacorogna, R. Davé, R. B. Olsen, O.V. Pictet, Journal of Empirical Finance, 4, 213 (1997).

[51] T. Lux, M. Marchesi, Nature 397, 498 (1999).

[52] I. Giardina, J.P. Bouchaud, M. Mézard, in preparation. 